TRANSACTIONS OF THE

AMERICAN MATHEMATICAL SOCIETY

Volume 354, Number 12, Pages 4953-4968

S 0002-9947(02)03063-5

Article electronically published on August 1, 2002

\title{
DETECTION OF RENEWAL SYSTEM FACTORS VIA THE CONLEY INDEX
}

\author{
JIM WISEMAN
}

\begin{abstract}
Let $N$ be an isolating neighborhood for a map $f$. If we can decompose $N$ into the disjoint union of compact sets $N_{1}$ and $N_{2}$, then we can relate the dynamics on the maximal invariant set $\operatorname{Inv} N$ to the shift on two symbols by noting which component of $N$ each iterate of a point $x \in \operatorname{Inv} N$ lies in. We examine a method, based on work by Mischaikow, Szymczak, et al., for using the discrete Conley index to detect explicit subshifts of the shift associated to $N$. In essence, we measure the difference between the Conley index of $\operatorname{Inv} N$ and the sum of the indices of $\operatorname{Inv} N_{1}$ and $\operatorname{Inv} N_{2}$.
\end{abstract}

\section{INTRODUCTION}

We begin with a continuous map of a locally compact metric space, $f: X \rightarrow X$ and an isolating neighborhood $N$ (i.e., $N$ is compact and the maximal invariant set of $N, \operatorname{Inv} N$, lies in the interior of $N$; see section 2 for precise definitions). If $N$ is the disjoint union of two other isolating neighborhoods $N_{1}$ and $N_{2}$, then we can associate a symbolic dynamical system to the map $f$ restricted to Inv $N$. We define (see [3]) a continuous map $\Theta: \operatorname{Inv} N \rightarrow\{1,2\}$ by setting

$$
\Theta(x)= \begin{cases}1 & \text { if } x \in N_{1}, \\ 2 & \text { if } x \in N_{2}\end{cases}
$$

Let $\left(\Sigma_{2}^{+}, \sigma\right)$ be the full one-sided shift on the symbols 1 and 2 . We relate the dynamics of $f$ on Inv $N$ to symbolic dynamics via the itinerary map $\rho: \operatorname{Inv} N \rightarrow \Sigma_{2}^{+}$ defined by

$$
\rho(x)=\left(\Theta(x), \Theta(f(x)), \Theta\left(f^{2}(x)\right), \ldots\right) .
$$

It is clear that $\rho$ is continuous and that $\sigma \circ \rho=\rho \circ f$.

We can use the discrete Conley index to detect interesting subshifts of the image shift $\rho(\operatorname{Inv} N)$. Roughly speaking, the Conley index assigns to each isolated invariant set a pointed space and a basepoint-preserving map. The method, based on work in 21] and 3], is to compare the induced homology maps on the pointed space for Inv $N$ to the direct sum of the maps on the spaces for Inv $N_{1}$ and $\operatorname{Inv} N_{2}$.

In [3], it is shown that if the eigenvalues of these various homology maps differ in a certain way, then there exists a positive integer $n$ such that $f^{n}$ factors onto the full two-shift $\left(\Sigma_{2}^{+}, \sigma\right)$. Similar results are applied to the Lorenz equations in [12]. The number $n$ is not specified, however; so this result provides no estimate for the

Received by the editors August 5, 2001 and, in revised form, March 28, 2002.

2000 Mathematics Subject Classification. Primary 37B30; Secondary 37B10, 54H20.

Key words and phrases. Conley index, symbolic dynamics, renewal system. 
topological entropy of $f$. Our main result, Theorem [5.6, avoids this difficulty by detecting explicit subshifts of $\rho(\operatorname{Inv} N)$, and when combined with the results in [12] shows that we can take $n=2$ for the Lorenz equations.

\section{The Discrete Conley index}

The discrete Conley index is a powerful topological tool for studying isolated invariant sets of a given map $f$. Roughly speaking, it assigns to each such set a pointed space $P$ and a base-point preserving map $f_{P}$, which is defined up to an equivalence relation. By studying the simpler map $f_{P}$ we can draw conclusions about the original map $f$.

The Conley index was originally developed for flows (see [5, 20]), and was later extended to the discrete-time case $([7,16,19,22])$. See [11] for a good introduction to the Conley index and its history.

An important feature of the discrete Conley index is that, under suitable hypotheses, if two maps $f$ and $g$ are $C^{0}$-close to each other, then they have the same index. Thus we can use it to obtain rigorous results from numerical approximations of, for example, Poincaré sections of flows arising from ODEs. (See [12, 14, 15, 17, 28.)

Our discussion of the discrete Conley index is based on that in [7, where one can find more details and proofs of the theorems below.

Let $U$ be an open subset of a locally compact metric space $X$ and suppose $f: U \rightarrow X$ is a continuous map.

Definition 2.1. For any set $N \subset U$ we define Inv $N$, the maximal invariant subset, to be the set of $x \in N$ such that there exists an orbit $\left\{x_{n}\right\}_{n \in \mathbb{Z}} \subset N$ with $x_{0}=x$ and $f\left(x_{n}\right)=x_{n+1}$ for all $n$. A compact set $N$ is called an isolating neighborhood if Inv $N \subset \operatorname{Int} N$. A set $S$ is called an isolated invariant set if there exists an isolating neighborhood $N$ with $S=\operatorname{Inv} N$. If $N$ is an isolating neighborhood, we define the exit set of $N$ to be

$$
N^{-}:=\{x \in N: f(x) \notin \operatorname{Int} N\} .
$$

Definition 2.2. Let $S$ be an isolated invariant set and suppose $L \subset N$ is a compact pair contained in the interior of the domain of $f$. The pair $(N, L)$ is called a filtration pair for $S$ provided $N$ and $L$ are each the closures of their interiors and

1. $\operatorname{cl}(N \backslash L)$ is an isolating neighborhood of $S$,

2. $L$ is a neighborhood of $N^{-}$in $N$, and

3. $f(L) \cap \operatorname{cl}(N \backslash L)=\emptyset$.

Theorem 2.3. Let $S$ be an isolated invariant set. For every neighborhood $V$ of $S$, there exists a filtration pair $(N, L)$ for $S$ with $L \subset N \subset V$. Moreover, there is a neighborhood of $f$ in the $C^{0}$ topology such that for any $\tilde{f}$ in this neighborhood, $\tilde{S}=\operatorname{Inv}(N \backslash L, \tilde{f})$ is an isolated invariant set and $(N, L)$ is a filtration pair for $\tilde{S}$.

Theorem 2.4. Let $P=(N, L)$ be a filtration pair for $f$ and let $N_{L}$ denote the quotient space $N / L$ where the collapsed set $L$ is denoted $[L]$ and is taken as the base-point. Then $f$ induces a continuous base-point preserving map $f_{P}: N_{L} \rightarrow N_{L}$ with the property $[L] \subset \operatorname{Int} f_{P}^{-1}([L])$.

Remark 2.5. Observe that we can identify the set $\operatorname{Inv}\left(N_{L}, f_{P}\right) \backslash\{[L]\}$ with $S=$ $\operatorname{Inv}(\operatorname{cl}(N \backslash L), f)$. 
Theorem 2.3 tells us that we can find a filtration pair for any isolated invariant set $S$. Our choice of filtration pairs is not unique, even up to homotopy equivalence. Any two filtration pairs for $S$ will, however, be shift equivalent, as we now discuss.

Suppose $\mathcal{K}$ is a category. Let $X, X^{\prime}$ be objects in $\mathcal{K}$ and $f: X \rightarrow X, g: X^{\prime} \rightarrow X^{\prime}$ be endomorphisms. We say that $(X, f)$ and $\left(X^{\prime}, g\right)$ are shift equivalent (a concept first described in [26]), or write $f \sim_{s} g$, if there exist $m \in \mathbb{Z}^{+}, r: X \rightarrow X^{\prime}$ and $s: X^{\prime} \rightarrow X$ such that the diagrams
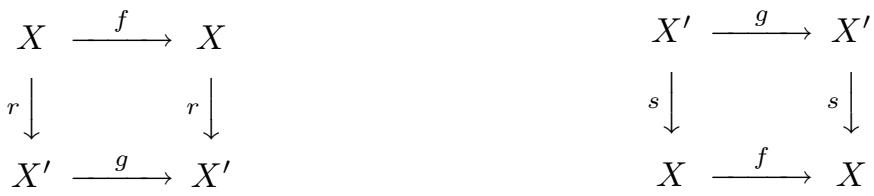

commute and $r \circ s=g^{m}$ and $s \circ r=f^{m}$. The integer $m$ is called the lag. We denote by $\llbracket f \rrbracket$ the shift equivalence class of $f$.

Again, see [7] for proofs of the theorems in this section.

Theorem 2.6. Suppose $P=(N, L)$ and $P^{\prime}=\left(N^{\prime}, L^{\prime}\right)$ are filtration pairs for $S$. Then the induced maps, $f_{P}$ and $f_{P^{\prime}}$, on the corresponding pointed spaces, are shift equivalent.

Let $S$ be an isolated invariant set, and consider the homotopy class of base-point preserving maps on $N_{L}$ with $f_{P}$ as a representative. We denote this collection $h_{P}(S)$. We may now make the following definition.

Definition 2.7. Let $S$ be an isolated invariant set for a continuous map $f$. Then define the discrete homotopy Conley index of $S, h(S)$, to be $\llbracket h_{P}(S) \rrbracket$, the shift equivalence class of $h_{P}(S)$, where $P=(N, L)$ is a filtration pair for $S$. We apply the singular homology functor (for the purposes of this paper, we will consider only real coefficients, i.e., in what follows $\left.H_{*}(-)=H_{*}(-; \mathbb{R})\right)$ to obtain the homological Conley index, $\mathrm{Con}_{*}(S)$. In other words, $\operatorname{Con}_{*}(S)$ is the shift equivalence class of $\left(f_{P}\right)_{*}: H_{*}\left(N_{L},[L]\right) \rightarrow H_{*}\left(N_{L},[L]\right)$ where $P=(N, L)$ is a filtration pair for $S$.

\section{RENEWAL SYSTEMS}

Let $\alpha=\left(\alpha_{0}, \alpha_{1}, \ldots, \alpha_{k}\right) \in\{1,2\}^{k+1}$ and $\beta=\left(\beta_{0}, \beta_{1}, \ldots, \beta_{l}\right) \in\{1,2\}^{l+1}$ be two finite words, and define $\Gamma(\alpha, \beta)$, the renewal system generated by $\alpha$ and $\beta$, to be the subshift of the full shift on the symbols 1 and 2 generated by all infinite concatenations of the words $\alpha$ and $\beta$. For example, $\Gamma((1),(2))$ is the full shift, and $\Gamma((1),(2,2))$ is the even shift. (In general, a renewal system (a generalization of subshifts of finite type introduced by Adler) can have more than two generating words (see [10, §13.1]), but we will be concerned primarily with those generated by only two.)

We are interested in the entropy of $\Gamma(\alpha, \beta)$. We first make the following definitions.

Definition 3.1. Define the finite word $\alpha * \beta$ by $\alpha * \beta:=\left(\alpha_{0}, \ldots, \alpha_{k}, \beta_{0}, \ldots, \beta_{l}\right)$. For $n$ a positive integer, define $n \cdot \alpha$ by setting $1 \cdot \alpha=\alpha$ and $n \cdot \alpha=\alpha *((n-1) \cdot \alpha)$. We say that two finite words $\omega^{1}$ and $\omega^{2}$ are independent if there do not exist a word $\alpha$ and positive integers $n_{1}$ and $n_{2}$ such that $\omega^{1}=n_{1} \cdot \alpha$ and $\omega^{2}=n_{2} \cdot \alpha$. 
It can be difficult in general to compute the entropy of a subshift, since it involves finding the roots of the characteristic polynomial of a (possibly large) related matrix. For a renewal system, however, it is much easier. (Thanks to Michał Misiurewicz for bringing the following to my attention.)

Theorem 3.2. Let $\alpha=\left(\alpha_{0}, \alpha_{1}, \ldots, \alpha_{k}\right)$ and $\beta=\left(\beta_{0}, \beta_{1}, \ldots, \beta_{l}\right)$ be two independent words. The topological entropy of $\Gamma(\alpha, \beta)$ is equal to the log of the absolute value of the largest root of the polynomial $X^{l+k-1}-X^{l-1}-X^{k-1}$.

Proof. This follows from the fact that the entropy is equal to the log of the spectral radius of a (suitably chosen) adjacency matrix (see [10, Ch. 4]), and from the very useful formula for the characteristic polynomial of such a matrix given in [1. Theorem 4.4.14].

If we don't want to go to the trouble of computing the entropy exactly, we can get an easy estimate by noting that if $\alpha$ and $\beta$ are independent, then the $N$ th power of $\Gamma(\alpha, \beta)$ (where $N$ is the least common multiple of $k$ and $l$ ) contains the full shift; so the entropy is at least $\frac{\log 2}{N}$. (Of course, if $\alpha$ and $\beta$ are not independent, then the entropy is zero.)

\section{The Conley indeX And Symbolic Dynamics}

The results in this section are essentially a restatement of those from [21] and [3] in less categorical language. Recall that $N$ is an isolating neighborhood that is the disjoint union of two other isolating neighborhoods, $N_{1}$ and $N_{2}$, and define the set $S$ to be $\operatorname{Inv} N$ and the sets $S_{i}$ to be Inv $N_{i}$ for $i=1,2$. We choose a filtration pair $P=(K, L)$ for $f$, with $K \subset N$. Then the homotopy Conley index of $S, h(S)$, is $\llbracket h_{P}(S) \rrbracket$, where we recall from section 2 that $f_{P}: K_{L} \rightarrow K_{L}$ is the induced map on the pointed space $K_{L}, h_{P}(S)$ is the homotopy class of basepoint-preserving maps of $K_{L}$ with $f_{P}$ as a representative, and $\llbracket-\rrbracket$ denotes the shift equivalence class.

For $i=1,2$, define $K_{i}:=K \cap N_{i}$ and $L_{i}:=L \cap N_{i}$. Note that $K_{L}=\left(K_{1}\right)_{L_{1}} \vee$ $\left(K_{2}\right)_{L_{2}}$ (where $\vee$ denotes the one-point union of pointed spaces). Unfortunately, the pair $\left(K_{i}, L_{i}\right)$ will not in general be a filtration pair for $S_{i}$. We can get around this problem, however.

Definition 4.1. Let $x$ be a point in $K$ and $[x]_{K_{L}}$ its image under the quotient map $K \rightarrow K_{L}$. Define the point $[x]_{\left(K_{i}\right)_{L_{i}}}$ similarly for $x$ in $\left(K_{i}\right)_{L_{i}}$. Define maps $r_{i}: K_{L} \rightarrow\left(K_{i}\right)_{L_{i}}$ by setting

$$
r_{i}\left([x]_{K_{L}}\right)= \begin{cases}{[x]_{\left(K_{i}\right)_{L_{i}}}} & \text { if } x \in K_{i} \backslash L_{i}, \\ {\left[L_{i}\right]_{\left(K_{i}\right)_{L_{i}}}} & \text { otherwise. }\end{cases}
$$

Also, let $e_{i}:\left(K_{i}\right)_{L_{i}} \hookrightarrow K_{L}=\left(K_{1}\right)_{L_{1}} \vee\left(K_{2}\right)_{L_{2}}$ be the inclusion map. Thus, if we consider $K_{L}$ as $\left(K_{1}\right)_{L_{1}} \vee\left(K_{2}\right)_{L_{2}}$, then $r_{1}$ simply collapses $\left(K_{2}\right)_{L_{2}}$ to the basepoint $\left[L_{1}\right]_{\left(K_{1}\right)_{L_{1}}}$ of $\left(K_{1}\right)_{L_{1}}$, and similarly for $r_{2}$. Also, $e_{1} \circ r_{1}: K_{L} \rightarrow K_{L}$ collapses $\left(K_{2}\right)_{L_{2}}$ to the basepoint $[L]_{K_{L}}$ of $K_{L}$, and similarly for $e_{2} \circ r_{2}$. Define a map $\tilde{f}_{P_{i}}: K_{L} \rightarrow K_{L}$ by setting $\tilde{f}_{P_{i}}:=f_{P} \circ e_{i} \circ r_{i}$, and define $h_{P_{i}}\left(S_{i}\right)$ to be the homotopy class of basepoint-preserving maps of $K_{L}$ with $\tilde{f}_{P_{i}}$ as a representative.

The point of all this notation is the following.

Theorem 4.2 ([23, Lemma 3.1]). In our notation, $h\left(S_{i}\right)=\llbracket h_{P_{i}}\left(S_{i}\right) \rrbracket$. 
Thus we can, in a sense, use the decompositions of the filtration pair $P=(K, L)$ for $S$ as filtration pairs $\left(K_{i}, L_{i}\right)$ for $S_{i}$, with the desirable property that $K_{L}=$ $\left(K_{1}\right)_{L_{1}} \vee\left(K_{2}\right)_{L_{2}}$.

Recall that $\operatorname{Con}_{*}(S)$ is the shift equivalence class of the map $\left(f_{P}\right)_{*}: H_{*}\left(K_{L},[L]_{K_{L}}\right)$ $\rightarrow H_{*}\left(K_{L},[L]_{K_{L}}\right)$ and, similarly, $\operatorname{Con}_{*}\left(S_{i}\right)=\llbracket\left(\tilde{f}_{P_{i}}\right)_{*} \rrbracket$. Note that since $K_{L}=$ $\left(K_{1}\right)_{L_{1}} \vee\left(K_{2}\right)_{L_{2}}$, we have that

$$
H_{*}\left(K_{L},[L]_{K_{L}}\right)=H_{*}\left(\left(K_{1}\right)_{L_{1}},\left[L_{1}\right]_{\left(K_{1}\right)_{L_{1}}}\right) \oplus H_{*}\left(\left(K_{2}\right)_{L_{2}},\left[L_{2}\right]_{\left(K_{2}\right)_{L_{2}}}\right) .
$$

Definition 4.3. Assume that for some positive integer $q, H_{q}\left(K_{L},[L]_{K_{L}}\right)$ is finitedimensional, and that $\operatorname{dim}\left(H_{q}\left(\left(K_{i}\right)_{L_{i}},\left[L_{i}\right]_{\left(K_{i}\right)_{L_{i}}}\right)\right) \geq 1$ for $i=1,2$. Let the set of vectors $\left(v_{1}, \ldots, v_{d_{1}}\right)$ be a basis for $H_{q}\left(\left(K_{1}\right)_{L_{1}},\left[L_{1}\right]_{\left(K_{1}\right)_{1}}\right)$, and $\left(v_{d_{1}+1}, \ldots, v_{d_{1}+d_{2}}\right)$ a basis for $H_{q}\left(\left(K_{2}\right)_{L_{2}},\left[L_{2}\right]_{\left(K_{2}\right)_{L_{2}}}\right)$. Furthermore, for $i, j=1$, 2, let $A_{i j}$ be the matrix representation for

$$
\left(r_{i} \circ f_{P} \circ e_{j}\right)_{q}: H_{q}\left(\left(K_{j}\right)_{L_{j}},\left[L_{j}\right]_{\left(K_{j}\right)_{j}}\right) \rightarrow H_{q}\left(\left(K_{i}\right)_{L_{i}},\left[L_{i}\right]_{\left(K_{i}\right)_{L_{i}}}\right) .
$$

Then

$$
\left(v_{1} \oplus 0, \ldots, v_{d_{1}} \oplus 0,0 \oplus v_{d_{1}+1}, \ldots, 0 \oplus v_{d_{1}+d_{2}}\right)
$$

is a basis for $H_{q}\left(K_{L},[L]_{K_{L}}\right)$ in which $\left(f_{P}\right)_{q}: H_{q}\left(K_{L},[L]_{K_{L}}\right) \rightarrow H_{q}\left(K_{L},[L]_{K_{L}}\right)$ has the matrix representation $A=\left(\begin{array}{l|l}A_{11} & A_{12} \\ \hline A_{21} & A_{22}\end{array}\right)$, where $A_{i j}$ is as above. Also, note that $\left(f_{P}\right)_{q}=\left(\tilde{f}_{P_{1}}\right)_{q}+\left(\tilde{f}_{P_{2}}\right)_{q}$. The matrix representations $A_{i}$ for $\left(\tilde{f}_{P_{i}}\right)_{q}$ are $A_{1}=\left(\begin{array}{l|l}A_{11} & 0 \\ \hline A_{21} & 0\end{array}\right)$ and $A_{2}=\left(\begin{array}{l|l}0 & A_{12} \\ \hline 0 & A_{22}\end{array}\right)$. (In what follows, we will occasionally abuse notation by using the matrices $A_{i j}$ and $A_{i}$ to denote the actual maps that they represent.)

Now, if the original map $f: X \rightarrow X$ causes no interactions between the sets $N_{1}$ and $N_{2}$ (i.e., if $f^{j}\left(N_{1}\right) \cap N_{2}=f^{j}\left(N_{2}\right) \cap N_{1}=\emptyset$ for all $j$ ), then $A_{12}$ and $A_{21}$ will both have only zero entries. In this case, the set $S$ will be equal to the disjoint union of $S_{1}$ and $S_{2}$, and the dynamics on $S$ will break into two pieces: those occurring on $S_{1}$ and those on $S_{2}$. In this case, the map $\rho$ will reflect no interesting symbolic dynamics, since $\rho(S)$ will be contained in the set $\{(1,1,1, \ldots)\} \cup\{(2,2,2, \ldots)\}$. Thus we will attempt to detect chaotic interaction between $N_{1}$ and $N_{2}$, in a sense, by measuring the failure of $A_{12}$ and $A_{21}$ to be zero.

A key to our analysis is the following property of the Conley index, for which a proof can be found in [7].

Theorem 4.4 (Ważewski property). Let $T$ be an isolated invariant set. If $h(T) \neq$ 0 , then $T$ is nonempty. In particular, if any of the induced homology maps is nonnilpotent, then $T$ is nonempty.

We will need a little more notation, borrowed from [3]. Given a word $\omega=$ $\left(\omega_{0}, \omega_{1}, \ldots, \omega_{k}\right) \in\{1,2\}^{k+1}$, define $N_{\omega}:=\bigcap_{j=0}^{k} f^{-j}\left(N_{\omega_{j}}\right)$ and $S_{\omega}:=\operatorname{Inv}\left(N_{\omega}, f^{k+1}\right)$. (Note that $N_{\omega}$ is an isolating neighborhood for $f^{k+1}$ with $S_{\omega}$ contained in $S$, as shown in 23.)

The following obvious proposition will be useful.

Proposition 4.5. The set $S_{\omega}$ is nonempty if and only if there is a point $x$ in $S$ such that $\rho(x)=\left(\omega_{0}, \omega_{1}, \ldots, \omega_{k}, \omega_{0}, \omega_{1}, \ldots, \omega_{k}, \omega_{0}, \ldots\right)$. 
Let $\left(f_{\omega}\right)_{q}$ be a representative of the map induced on $\operatorname{Con}_{q}\left(S_{\omega}\right)$ by $f^{k+1}$, and define the matrix $\left(A_{*}\right)_{\omega}$ by setting

$$
\left(A_{*}\right)_{\omega}:=\left(A_{\omega_{k}} \circ A_{\omega_{k-1}} \circ A_{\omega_{k-2}} \circ \cdots \circ A_{\omega_{1}} \circ A_{\omega_{0}}\right) .
$$

Then Lemma 3.1 in 23 gives us the following.

Theorem 4.6. For any finite word $\omega, \llbracket\left(f_{\omega}\right)_{q} \rrbracket=\llbracket\left(A_{*}\right)_{\omega} \rrbracket$.

Let us define the matrix $\left(A_{* *}\right)_{\omega}$ by setting

$$
\left(A_{* *}\right)_{\omega}:=\left(A_{\omega_{0} \omega_{k}} \circ A_{\omega_{k} \omega_{k-1}} \circ A_{\omega_{k-1} \omega_{k-2}} \circ \cdots \circ A_{\omega_{2} \omega_{1}} \circ A_{\omega_{1} \omega_{0}}\right) .
$$

Note that $\left(A_{* *}\right)_{\omega}$ and $\left(A_{*}\right)_{\omega}$ are not, in general, equal, but they are shift equivalent, as the following theorem shows.

Theorem 4.7. For any finite word $\omega$, $\left(A_{* *}\right)_{\omega} \rrbracket=\llbracket\left(A_{*}\right)_{\omega} \rrbracket$.

Proof. Let us assume (without loss of generality) that $\omega_{0}=1$. It is easy to see by direct computation (using the fact that $A_{i j}$ represents the map $\left(r_{i} \circ f_{P} \circ e_{j}\right)_{q}$ and $A_{i}$ the map $\left.\left(f_{P} \circ e_{i} \circ r_{i}\right)_{q}\right)$ that in this case,

$$
\left(A_{*}\right)_{\omega}=\left(\begin{array}{c|c}
\left(A_{* *}\right)_{\omega} & 0 \\
\hline M & 0
\end{array}\right)
$$

where $M$ is some matrix that represents a map from $H_{q}\left(\left(K_{1}\right)_{L_{1}},\left[L_{1}\right]_{\left(K_{1}\right)_{L_{1}}}\right)$ to $H_{q}\left(\left(K_{2}\right)_{L_{2}},\left[L_{2}\right]_{\left(K_{2}\right)_{L_{2}}}\right.$ ) (we could determine $M$ explicitly, but we will see that it does not matter exactly what it is). Since $\left(A_{* *}\right)_{\omega}$ is clearly shift equivalent to the map from $H_{q}\left(K_{L},[L]_{K_{L}}\right)$ to itself represented by the matrix $\left(\begin{array}{c|c}\left(A_{* *}\right)_{\omega} & 0 \\ \hline 0 & 0\end{array}\right)$ (using the natural inclusion and projection maps of $H_{q}\left(\left(K_{1}\right)_{L_{1}},\left[L_{1}\right]_{\left(K_{1}\right)_{L_{1}}}\right)$ as a subspace of $\left.H_{q}\left(K_{L},[L]_{K_{L}}\right)=H_{q}\left(\left(K_{1}\right)_{L_{1}},\left[L_{1}\right]_{\left(K_{1}\right)_{L_{1}}}\right) \oplus H_{q}\left(\left(K_{2}\right)_{L_{2}},\left[L_{2}\right]_{\left(K_{2}\right)_{L_{2}}}\right)\right)$, the theorem is an immediate consequence of the following lemma.

Lemma 4.8. The map $F: V \rightarrow V$ represented by the matrix $\left(\frac{C}{D} \mid 0\right)$ is shift equivalent to the map $G: V \rightarrow V$ represented by the matrix $\left(\begin{array}{l|l}C & 0 \\ \hline 0 & 0\end{array}\right)$, where $V=$ $V_{1} \oplus V_{2}$ is a finite-dimensional vector space and $C$ is a matrix representing a map from $V_{1}$ to itself.

Proof. It is easy to verify that the maps $r, s: V \rightarrow V$, where $s$ is represented by the matrix $\left(\begin{array}{l|l}C & 0 \\ \hline D & 0\end{array}\right)$ and $r$ by the matrix $\left(\begin{array}{c|c}\operatorname{Id}_{V_{1}} & 0 \\ \hline 0 & 0\end{array}\right)$, generate a shift equivalence of lag one between $F$ and $G$. (See section [2)

Corollary 4.9. For any finite word $\omega, \llbracket\left(f_{\omega}\right)_{q} \rrbracket=\llbracket\left(A_{* *}\right)_{\omega} \rrbracket=\llbracket\left(A_{*}\right)_{\omega} \rrbracket$.

Proof. This follows from Theorems 4.6 and 4.7 and the fact that shift equivalence is an equivalence relation.

In other words, Corollary 4.9 tells us that once we know the matrix $A=$ $\left(\begin{array}{l|l}A_{11} & A_{12} \\ \hline A_{21} & A_{22}\end{array}\right)$ for the representative of the map on $\operatorname{Con}_{q}(S)$, we can find a representative $\left(A_{*}\right)_{\omega}$ (or $\left.\left(A_{* *}\right)_{\omega}\right)$ for the map on $\operatorname{Con}_{q}\left(S_{\omega}\right)$ by simply concatenating the pieces $A_{i}$ (or $A_{i j}$ ) of $A$ in the appropriate order.

Remark 4.10. Thus, to show that $S_{\omega}$ is nonempty, it is enough, by the Ważewski property, to show that the matrix $\left(A_{*}\right)_{\omega}$ is nonnilpotent (or, equivalently, that $\left(A_{* *}\right)_{\omega}$ is nonnilpotent). (The equivalence follows from the fact that any matrix shift-equivalent to a nilpotent matrix is itself nilpotent.) 
We will use this observation to hunt for renewal systems. For $i=1,2$, let $k_{i}$ be a nonnegative integer and $\omega^{i}=\left(\omega_{0}^{i}, \ldots, \omega_{k_{i}}^{i}\right)$ an element of $\{1,2\}^{k_{i}+1}$. Also, let $\alpha=\left(\alpha_{0}, \alpha_{1}, \ldots\right)$ be an element of $\{1,2\}^{l}$, where $l$ is in $\mathbb{N} \cup\{+\infty\}$. Define the sequence $\alpha\left(\omega^{1}, \omega^{2}\right)$ (which will be infinite if $\alpha$ is infinite) by setting

$$
\begin{aligned}
\alpha\left(\omega^{1}, \omega^{2}\right) & :=\left(\left(\omega^{\alpha_{0}}\right),\left(\omega^{\alpha_{1}}\right),\left(\omega^{\alpha_{2}}\right), \ldots\right) \\
& =\left(\omega_{0}^{\alpha_{0}}, \ldots, \omega_{k_{\alpha_{0}}}^{\alpha_{0}}, \omega_{0}^{\alpha_{1}}, \ldots, \omega_{k_{\alpha_{1}}}^{\alpha_{1}}, \omega_{0}^{\alpha_{2}}, \ldots\right) .
\end{aligned}
$$

Thus, the word $\alpha\left(\omega^{1}, \omega^{2}\right)$ is a concatenation of the words $\omega^{1}$ and $\omega^{2}$, combined in the order specified by $\alpha$.

Note that if $\alpha$ is finite and equal to $\left(\alpha_{0}, \ldots, \alpha_{l}\right)$, then by definition,

$$
\left(A_{*}\right)_{\alpha\left(\omega^{1}, \omega^{2}\right)}=\left(A_{*}\right)_{\omega^{\alpha_{l}}} \circ\left(A_{*}\right)_{\omega^{\alpha} l-1} \circ \cdots \circ\left(A_{*}\right)_{\omega^{\alpha_{1}}} \circ\left(A_{*}\right)_{\omega^{\alpha_{0}}} .
$$

Also, note that the equation

$$
\left(A_{* *}\right)_{\alpha\left(\omega^{1}, \omega^{2}\right)}=\left(A_{* *}\right)_{\omega^{\alpha_{l}}} \circ\left(A_{* *}\right)_{\omega^{\alpha_{l-1}}} \circ \cdots \circ\left(A_{* *}\right)_{\omega^{\alpha_{1}}} \circ\left(A_{* *}\right)_{\omega^{\alpha_{0}}}
$$

does not hold in general (for example, $\left(A_{* *}\right)_{12} \neq A_{22} \circ A_{11}$ ). However, it does hold if $\omega_{0}^{1}=\omega_{0}^{2}$. Since we wish to consider products of $\left(A_{* *}\right)_{\omega}$ 's as on the right side of equation (3), we will, in general, consider only the case $\omega_{0}^{1}=\omega_{0}^{2}$ when discussing $\left(A_{* *}\right)_{\alpha\left(\omega^{1}, \omega^{2}\right)}$.

Remark 4.11. By Theorem 4.7, $\left(A_{*}\right)_{\omega}$ is nilpotent if and only if $\left(A_{* *}\right)_{\omega}$ is. Therefore, in what follows, we will for the sake of simplicity state conditions involving only $\left(A_{*}\right)_{\omega}$ 's nilpotence, leaving unstated the similar conditions on $\left(A_{* *}\right)_{\omega}$.

Definition 4.12. Let $\omega=\left(\omega_{0}, \omega_{1}, \ldots, \omega_{k}\right)$ be a finite word. Define $l(\omega)$, the length of $\omega$, to be $k+1$.

Theorem 4.13. Suppose that $\omega^{1}$ and $\omega^{2}$ are two words such that $\left(A_{*}\right)_{\alpha\left(\omega^{1}, \omega^{2}\right)}$ is nonnilpotent for every finite $\alpha$. Then for any $\beta=\left(\beta_{0}, \beta_{1}, \ldots\right) \in\{1,2\}^{+\infty}$ there exists a point $x \in S$ such that $\rho(x)=\beta\left(\omega^{1}, \omega^{2}\right)$.

Another way to state the conclusion of this theorem is that the renewal system $\Gamma\left(\omega^{1}, \omega^{2}\right)$ generated by all infinite concatenations of the blocks $\omega^{1}$ and $\omega^{2}$ is contained in the image of $S$ under $\rho$.

Proof. If the word $\alpha$ is periodic, this follows from Theorem 4.4. Proposition 4.5. and Corollary 4.9 In general, we write $\alpha$ as the limit of periodic words. See section 4 of [3] for details.

\section{Method FOR Detection}

Given two matrices $A_{1}$ and $A_{2}$, the set of infinite sequences $\beta$ such that the matrix

$$
\left(A_{*}\right)_{\beta_{j}} \circ\left(A_{*}\right)_{\beta_{j-1}} \circ \cdots \circ\left(A_{*}\right)_{\beta_{0}}
$$

is nonnilpotent for every integer $j \geq 0$, along with the shift map $\sigma$, is an example of a cocyclic subshift, a class of subshifts which properly generalizes sofic shifts, which themselves generalize subshifts of finite type. See [9] for a complete account. In general, cocyclic subshifts can be quite complicated.

We wish to reduce the problem to something simpler. In light of Theorem 4.13 and equations (2) and (3), the obvious question to ask is how to find words $\omega^{1}$ and $\omega^{2}$ such that any finite concatenation of the corresponding matrices $\left(A_{*}\right)_{\omega^{1}}$ and $\left(A_{*}\right)_{\omega^{2}}$ (or of $\left(A_{* *}\right)_{\omega^{1}}$ and $\left(A_{* *}\right)_{\omega^{2}}$ if $\left.\omega_{0}^{1}=\omega_{0}^{2}\right)$ is nonnilpotent. That is, rather 
than studying the set of all solutions (the cocylic subshift), we will be satisfied if we can actually produce $\omega^{1}$ and $\omega^{2}$ as above in order to find a semiconjugacy onto a renewal system, which is simpler and easier to analyze in general than a cocyclic shift. We will see that our approach reduces essentially to the following situation.

We have two $n \times n$ matrices $M_{1}$ and $M_{2}$. Again, ideally we would like to know exactly the set $\mathcal{A}\left(M_{1}, M_{2}\right)$ of words $\beta \in\{1,2\}^{+\infty}$ such that the matrix

$$
M_{\beta_{j}} \circ M_{\beta_{j-1}} \circ \cdots \circ M_{\beta_{0}}
$$

is nonnilpotent for all nonnegative integers $j$. If the matrices are small enough, we may be able to compute $\mathcal{A}\left(M_{1}, M_{2}\right)$ explicitly. This question may be quite difficult to answer in general, however ([9] is devoted to this topic). A simpler question is whether or not $\mathcal{A}\left(M_{1}, M_{2}\right)$ is equal to the set $\{1,2\}^{+\infty}$, i.e., whether every finite concatenation of $M_{1}$ and $M_{2}$ is nonnilpotent. One might hope that we could answer this question in general by examining some bounded number of products, where the bound depended only on the size $n$ of the matrices. However, this is not the case, as shown by the matrices $M_{1}=\left(\begin{array}{ll}2 & 0 \\ 0 & 1\end{array}\right)$ and $M_{2}=\left(\begin{array}{ll}1 & -2^{m} \\ 1 & -2^{m}\end{array}\right)$, where $m$ is an arbitrary positive integer (the shortest nilpotent product is $M_{1}^{m} M_{2}$ ).

So we will try yet another question: given two $n \times n$ matrices $M_{1}$ and $M_{2}$, can we find conditions guaranteeing that any finite product of $M_{1}$ and $M_{2}$ will have rank greater than or equal to some $r>0$ ? (Note that if every finite product has rank at least $r$, then every finite product is nonnilpotent.) The advantage of this question is that we can answer it.

Definition 5.1. Let $m$ be a positive integer. We say that two $n \times n$ matrices $M_{1}$ and $M_{2}$ are $m$-compatible if there exists a positive integer $r$ such that

$$
\operatorname{rk}\left(M_{1} \circ\left(M_{i}\right)_{\omega}\right)=\operatorname{rk}\left(M_{2} \circ\left(M_{i}\right)_{\omega}\right)=\operatorname{rk}\left(\left(M_{i}\right)_{\omega}\right)=r
$$

for all words $\omega$ of length $m$.

Lemma 5.2. Let $M_{1}$ and $M_{2}$ be a pair of $m$-compatible matrices. Then $\left(M_{i}\right)_{\alpha}$ is nonnilpotent for any finite word $\alpha$.

Proof. In fact, $\left(M_{i}\right)_{\alpha}$ will have rank at least $r$. If $\alpha$ has length less than or equal to $m$, then it is a subword of a word $\omega$ of length $m$. Since by hypothesis $\operatorname{rk}\left(\left(M_{i}\right)_{\omega}\right)=r$, $\operatorname{rk}\left(\left(M_{i}\right)_{\alpha}\right) \geq r$.

Now suppose that $\alpha=\left(\alpha_{0}, \ldots, \alpha_{m+k}\right)(k \geq 0)$, and define $\alpha^{\prime}=\left(\alpha_{k+1}, \ldots, \alpha_{m+k}\right)$. It suffices to show that

$$
\operatorname{im}\left(\left(M_{i}\right)_{\alpha}\right)\left(=\operatorname{im}\left(\left(M_{i}\right)_{\alpha_{m+k}} \circ\left(M_{i}\right)_{\alpha_{m+k-1}} \circ \cdots \circ\left(M_{i}\right)_{\alpha_{0}}\right)\right)=\operatorname{im}\left(\left(M_{i}\right)_{\alpha^{\prime}}\right),
$$

since $\operatorname{dim}\left(\operatorname{im}\left(\left(M_{i}\right)_{\alpha^{\prime}}\right)\right)=r$. This follows by induction on $k$ (the case $k=0$ is exactly the condition of being $m$-compatible).

Remark 5.3. The converse is not true, as we see by taking $M_{1}=\left(\begin{array}{ll}1 & 0 \\ 0 & 1\end{array}\right)$ and $M_{2}=$ $\left(\begin{array}{ll}1 & 0 \\ 0 & 0\end{array}\right)$.

We will consider primarily the case $m=1$, since the computations are simpler; in this case, we say simply that $M_{1}$ and $M_{2}$ are compatible. (Note that two matrices are compatible if and only if they and their four pairwise products all have the same nonzero rank.) We say that two finite words $\alpha$ and $\beta$ are $\left(A_{*}\right)$-compatible if $\left(A_{*}\right)_{\alpha}$ and $\left(A_{*}\right)_{\beta}$ are compatible. Finally, we say that $\alpha$ and $\beta$ are $\left(A_{* *}\right)$-compatible if $\alpha_{0}=\beta_{0}$ and $\left(A_{* *}\right)_{\alpha}$ and $\left(A_{* *}\right)_{\beta}$ are compatible. 
Of course, any nonnilpotent matrix is $m$-compatible with itself for some $m$, but this case is not interesting. We will be interested primarily in independent, $\left(A_{*}\right)$-compatible words. We do not lose too much information by considering only compatibility, rather than $m$-compatibility or nonnilpotence more generally, as the following lemma shows.

Lemma 5.4. Let $\omega^{1}$ and $\omega^{2}$ be independent finite words such that any finite product of $\left(A_{*}\right)_{\omega^{1}}$ and $\left(A_{*}\right)_{\omega^{2}}$ has rank at least $r$ for some $r>0$. Let $R=\max _{k=1,2} \operatorname{rk}\left(A_{*}\right)_{\omega^{k}}$ and $L=\max _{k=1,2} l\left(\omega^{k}\right)$, the greater of the lengths of $\omega^{1}$ and $\omega^{2}$. Then there exist two independent $\left(A_{*}\right)$-compatible words $\bar{\omega}^{1}$ and $\bar{\omega}^{2}$ of length less than or equal to $\left(2^{R-r+1}-1\right) L$ which are concatenations of $\omega^{1}$ and $\omega^{2}$.

Proof. To show that there are two such $\left(A_{*}\right)$-compatible words, it would be enough to take $\bar{\omega}^{1}$ and $\bar{\omega}^{2}$ to be any two independent concatenations of $\omega^{1}$ and $\omega^{2}$ of minimal rank. However, it is easy to see that there is no upper bound on the minimum length of such words.

Instead, we will explicitly construct $\bar{\omega}^{1}$ and $\bar{\omega}^{2}$ by concatenating $\omega^{1}$ and $\omega^{2}$. The following lemma is the key to the construction. To simplify notation, we will for the rest of this proof use " $\operatorname{rk}(\alpha)$ " to denote " $\operatorname{rk}\left(\left(A_{*}\right)_{\alpha}\right)$," where $\alpha$ is any finite word.

Lemma 5.5. Let $\alpha$ and $\beta$ be two finite concatenations of the given words $\omega^{1}$ and $\omega^{2}$. Let $R^{\prime}=\max (\operatorname{rk}(\alpha), \operatorname{rk}(\beta))$ and $L^{\prime}=\max (l(\alpha), l(\beta))$. If $\alpha$ and $\beta$ are not $\left(A_{*}\right)$-compatible, we can find two independent words $\gamma$ and $\delta$ which are finite concatenations of $\omega^{1}$ and $\omega^{2}$ such that $\max (\operatorname{rk}(\gamma), \operatorname{rk}(\delta)) \leq R^{\prime}-1$ and $\max (l(\gamma), l(\delta)) \leq 2 L^{\prime}+L$.

Proof of Lemma 5.5. Since $\alpha$ and $\beta$ are not $\left(A_{*}\right)$-compatible, at least one of the four pairwise products $\alpha * \alpha, \alpha * \beta, \beta * \alpha$, and $\beta * \beta$ has rank strictly less than $R^{\prime}$. Let $\gamma$ be one such product. If $\gamma=n \cdot \omega^{1}$ for some integer $n$, let $\delta=\omega^{2} * \gamma$; otherwise, let $\delta=\omega^{1} * \gamma$. In either case, $\operatorname{rk}(\delta) \leq \operatorname{rk}(\gamma)<R^{\prime}$. Also, $l(\gamma) \leq 2 L^{\prime}$, and so $l(\delta) \leq 2 L^{\prime}+L$.

Of course, if $\omega^{1}$ and $\omega^{2}$ are $\left(A_{*}\right)$-compatible, we do not have to do anything. Otherwise, we apply the above lemma to them. If the resulting words $\gamma$ and $\delta$ are $\left(A_{*}\right)$-compatible, we are done. Otherwise, we apply the lemma again, this time to $\gamma$ and $\delta$. Since every concatenation of $\omega^{1}$ and $\omega^{2}$ has rank at least $r$, we will have to apply the lemma at most $R-r$ times; so the length of the resulting words will be at most

$$
\begin{aligned}
& \overbrace{2(\ldots 2(2}^{R-r} L+L)+L)+L \ldots)+L \\
& \quad=2^{R-r} L+2^{R-r-1} L+\cdots+2 L+L=\left(2^{R-r+1}-1\right) L .
\end{aligned}
$$

Theorem 5.6 (Main Theorem). Let $A$ be the Conley index homology map from Definition 4.3. If two words $\omega^{1}$ and $\omega^{2}$ are $\left(A_{*}\right)$ - or $\left(A_{* *}\right)$-compatible, then $\rho(S)$, the symbolic dynamics associated to the isolated invariant set $S$, contains the renewal system $\Gamma\left(\omega^{1}, \omega^{2}\right)$.

Proof. This follows from Theorem 4.13, Lemma 5.2, and equations (2) and (3) (in section 4). 
The method is now easy to state. We look at the set of all finite words and try to find two independent words $\omega^{1}$ and $\omega^{2}$ that are $\left(A_{*}\right)$-compatible (or, if $\omega_{0}^{1}=\omega_{0}^{2}$, $\left(A_{* *}\right)$-compatible). If we find two such words, then Theorem 5.6 tells us that the renewal system $\Gamma\left(\omega^{1}, \omega^{2}\right)$ generated by all infinite concatenations of $\omega^{1}$ and $\omega^{2}$ is contained in the image of $S$ under $\rho$. (We could consider dependent words as well, but the renewal system that they generate is not very interesting. If $\omega^{i}=n_{i} \cdot \alpha$ for $i=1,2$, then $\Gamma$ consists only of infinite repetitions of the word $\alpha$, and has topological entropy equal to zero. Still, the fact that there is a point $x \in S$ with itinerary $\rho(x)=(\alpha, \alpha, \alpha, \ldots)$ could be of interest. $)$

One way to implement this method is as follows. For $i=1,2$ and $n$ a positive integer, define $W(i, n)$ to be the set of words $\omega$ of length less than or equal to $n$ with $\omega_{0}=i$. Look at $W(1,2)$ and compute the rank of $\left(A_{* *}\right)_{\omega}$ for each $\omega$ in the set. Then, for each pair of independent words with the same nonzero rank, compute the rank of their four pairwise products. If all four have the same rank as the original two, then we are done. Otherwise, we keep looking. If we do not find anything in $W(1,2)$, look at $W(2,2)$, then expand the search to $W(i, 3)$, $W(i, 4)$, and so on, until we find something or give up. At the same time, we search $W(n):=W(1, n) \cup W(2, n)$ (the set of all words of length less than or equal to $n$ ) for a pair of $\left(A_{*}\right)$-compatible words. One advantage of this method is that it can easily be implemented on a computer.

There are a few tricks that we can use to speed up the process. First, we need the following definition, borrowed from [3].

Definition 5.7. Note that if $M$ is an $n \times n$ matrix, there is an integer $K \leq n$ such that for any $k \geq K, \operatorname{rk}\left(M^{k}\right)=\operatorname{rk}\left(M^{K}\right)$. The asymptotic rank of $M$ is defined by $\operatorname{ark}(M)=\operatorname{rk}\left(\bar{M}^{K}\right)$.

Now we notice that the matrices associated to the words $\alpha$ and $n \cdot \alpha$ have the same asymptotic rank. Thus, if $\operatorname{rk}\left(\left(A_{*}\right)_{\alpha}\right)=\operatorname{ark}\left(\left(A_{*}\right)_{\alpha}\right)$, then the same is true for $\left(A_{*}\right)_{n \cdot \alpha}$, and, in fact, the two matrices have the same image. So, if $\beta$ is another finite word, $\alpha$ and $\beta$ are $\left(A_{*}\right)$-compatible if and only if $n \cdot \alpha$ and $\beta$ are. Therefore, once we have such an $\alpha$, we do not need to check $n \cdot \alpha$ for any $n$. (Similar statements hold for $\left(A_{* *}\right)_{\alpha}$ and $\left.\left(A_{* *}\right)_{n \cdot \alpha \cdot}\right)$

If $\alpha=\left(\alpha_{0}, \ldots, \alpha_{n}\right)$, define the word $\tau(\alpha)$ to be $\left(\alpha_{n}, \alpha_{0}, \ldots, \alpha_{n-1}\right)$. Observe that for any integer $k \geq 2$, we have that

$$
\operatorname{rk}\left(\left(A_{*}\right)_{\alpha}^{k}\right)=\operatorname{rk}\left(\left(A_{*}\right)_{k \cdot \alpha}\right) \leq \operatorname{rk}\left(\left(A_{*}\right)_{\tau(\alpha)}^{(k-1)}\right)
$$

since $(k-1) \cdot \tau(\alpha)$ is a subword of $k \cdot \alpha$. Similarly,

$$
\operatorname{rk}\left(\left(A_{*}\right)_{\tau(\alpha)}^{k}\right)=\operatorname{rk}\left(\left(A_{*}\right)_{k \cdot \tau(\alpha)}\right) \leq \operatorname{rk}\left(\left(A_{*}\right)_{\alpha}^{(k-1)}\right),
$$

since $(k-1) \cdot \alpha$ is a subword of $k \cdot \tau(\alpha)$. Thus, $\operatorname{ark}\left(\left(A_{*}\right)_{\alpha}\right)=\operatorname{ark}\left(\left(A_{*}\right)_{\tau(\alpha)}\right)$. (Again, similar statements hold for $\left(A_{* *}\right)_{\alpha}$ and $\left(A_{* *}\right)_{\tau(\alpha)}$.)

It is also true that we do not need to check every pair of words for both $\left(A_{*}\right)$ and $\left(A_{* *}\right)$-compatibility.

Theorem 5.8. Let $\omega^{1}$ and $\omega^{2}$ be finite words such that $\omega_{0}^{1}=\omega_{0}^{2}$. If $\omega^{1}$ and $\omega^{2}$ are $\left(A_{*}\right)$-compatible, then they are $\left(A_{* *}\right)$-compatible.

If $\omega^{1}$ and $\omega^{2}$ are $\left(A_{* *}\right)$-compatible, then there exist positive integers $n_{k}(k=1$, 2) with $n_{k} \leq \operatorname{rk}\left(\left(A_{*}\right)_{\omega^{k}}\right)$ such that $n_{1} \cdot \omega^{1}$ and $n_{2} \cdot \omega^{2}$ are $\left(A_{*}\right)$-compatible. 
Proof. This follows from the definitions of compatibility and asymptotic rank and the following lemma.

Lemma 5.9. If $\alpha$ is any finite word, then $\operatorname{rk}\left(\left(A_{*}\right)_{\alpha}\right) \geq \operatorname{rk}\left(\left(A_{* *}\right)_{\alpha}\right)$, and $\operatorname{ark}\left(\left(A_{*}\right)_{\alpha}\right)$ $=\operatorname{ark}\left(\left(A_{* *}\right)_{\alpha}\right)$.

Proof. The statement about the ranks follows from equation (11) in section 4. To prove the statement about the asymptotic ranks, notice that all of the maps in the proofs of Theorem 4.7 and Lemma 4.8 are linear. Thus, we can find linear maps $r$ and $s$ that generate a shift equivalence of lag $m$ between $\left(A_{* *}\right)_{\alpha}$ and $\left(A_{*}\right)_{\alpha}$ (where $m$ is some positive integer). Let $n$ be any integer sufficiently large such that for any $l \geq n, \operatorname{rk}\left(\left(A_{*}\right)_{\alpha}^{l}\right)=\operatorname{ark}\left(\left(A_{*}\right)_{\alpha}\right)$ and $\operatorname{rk}\left(\left(A_{* *}\right)_{\alpha}^{l}\right)=\operatorname{ark}\left(\left(A_{* *}\right)_{\alpha}\right)$. Observe that

$$
\left(A_{* *}\right)_{\alpha}^{m+n}=s \circ r \circ\left(A_{* *}\right)_{\alpha}^{n}=s \circ\left(A_{*}\right)_{\alpha}^{n} \circ r
$$

so

$$
\operatorname{rk}\left(\left(A_{* *}\right)_{\alpha}^{m+n}\right)=\operatorname{ark}\left(\left(A_{* *}\right)_{\alpha}\right) \leq \operatorname{rk}\left(\left(A_{*}\right)_{\alpha}^{n}\right)=\operatorname{ark}\left(\left(A_{*}\right)_{\alpha}\right)
$$

Similarly,

$$
\left(A_{*}\right)_{\alpha}^{m+n}=r \circ s \circ\left(A_{*}\right)_{\alpha}^{n}=r \circ\left(A_{* *}\right)_{\alpha}^{n} \circ s
$$

so

$$
\operatorname{rk}\left(\left(A_{*}\right)_{\alpha}^{m+n}\right)=\operatorname{ark}\left(\left(A_{*}\right)_{\alpha}\right) \leq \operatorname{rk}\left(\left(A_{* *}\right)_{\alpha}^{n}\right)=\operatorname{ark}\left(\left(A_{* *}\right)_{\alpha}\right) .
$$

$\operatorname{Thus} \operatorname{ark}\left(\left(A_{* *}\right)_{\alpha}\right)=\operatorname{ark}\left(\left(A_{*}\right)_{\alpha}\right)$.

So we see that any renewal system detected by examining the $\left(A_{*}\right)_{\omega}$ 's is also detected by the $\left(A_{* *}\right)_{\omega}$ 's, but the $\left(A_{*}\right)_{\omega}$ 's may detect only a subshift of a shift detected by the $\left(A_{* *}\right)_{\omega}$ 's. Also, since the $\left(A_{* *}\right)_{\omega}$ 's act on a lower-dimensional space than do the $\left(A_{*}\right)_{\omega}$ 's, the computations with the former will be easier.

However, $\omega^{1}$ and $\omega^{2}$ can be $\left(A_{* *}\right)$-compatible only if $\omega_{0}^{1}=\omega_{0}^{2}$. Thus, for example, we cannot detect the shift generated by (1) and (2) using the $\left(A_{* *}\right)_{\omega}$ 's, but we might be able to using the $\left(A_{*}\right)_{\omega}$ 's. What we should do, then, given $\omega^{1}$ and $\omega^{2}$, is to check for $\left(A_{* *}\right)$-compatibility if $\omega_{0}^{1}=\omega_{0}^{2}$, and check for $\left(A_{*}\right)$-compatibility only if $\omega_{0}^{1} \neq \omega_{0}^{2}$.

Of course, for a given matrix $A$, it may well be that there simply are no compatible words (the case where $A$ is a matrix with only zero entries is a trivial example). So, no matter how long we look, we will not find any. This leads to the following question.

Question 5.10. Is there a number $N$, possibly depending on the dimension of $H_{q}\left(\left(K_{i}\right)_{L_{i}},\left[L_{i}\right]_{\left(K_{i}\right)_{L_{i}}}\right)$, such that if there are no $\left(A_{* *}\right)$-compatible (respectively $\left(A_{*}\right)$ compatible) words for our method in $W(i, N)$ (respectively $W(N)$ ), then there are no such words in $W(i, n)$ (respectively $W(n))$ for any $n$ ?

We have only partial answers to this question. The first is based on a result in [18], and is essentially a sharpening of Proposition 3.3 of [3].

Recall that $\operatorname{dim}\left(H_{q}\left(\left(K_{i}\right)_{L_{i}},\left[L_{i}\right]_{\left(K_{i}\right)_{L_{i}}}\right)\right)=d_{i}$, and let $d=d_{1}+d_{2}=\operatorname{dim}\left(H_{q}\left(K_{L}\right.\right.$, $\left.\left.[L]_{K_{L}}\right)\right)$.

Theorem 5.11. Let $\left\{\omega^{k}\right\}$ be a collection of $2^{\left(\begin{array}{c}d_{i} \\ r\end{array}\right)}$ finite words such that for each $\omega^{k}$ in the set, $\omega_{0}^{k}=i$ and $\operatorname{rk}\left(\left(A_{* *}\right)_{\omega^{k}}\right)=\operatorname{ark}\left(\left(A_{*_{*}}\right)_{\omega^{k}}\right)=r$. Then $\left\{\omega^{k}\right\}$ contains at least one pair of $\left(A_{* *}\right)$-compatible words. 
Similarly, if $\left\{\omega^{l}\right\}$ is a collection of $2^{\left(\begin{array}{c}d \\ r\end{array}\right)}$ finite words such that $\operatorname{rk}\left(\left(A_{*}\right)_{\omega^{l}}\right)=$ $\operatorname{ark}\left(\left(A_{*}\right)_{\omega^{l}}\right)=r$ for each $\omega^{l}$, then $\left\{\omega^{l}\right\}$ contains at least one pair of $\left(A_{*}\right)$-compatible words.

This theorem tells us that if we keep finding independent nonnilpotent words, we will eventually find some that are compatible.

Proof. Notice that a linear map $M$ on an $n$-dimensional vector space $V$ has rank $r$ if and only if $M^{\wedge r}: \Lambda^{r}(V) \rightarrow \Lambda^{r}(V)$ (the induced map on the $r$ th exterior power of $V)$ has rank one. This allows us to reduce everything to the rank-one case. Since $\operatorname{dim}\left(\Lambda^{r}(V)\right)=\left(\begin{array}{l}n \\ r\end{array}\right)$ (see [25, Ch. 2]), the theorem is a consequence of the following lemma.

Lemma 5.12. Let $V$ be an $n$-dimensional vector space, and let $\mathcal{M}=\left\{M_{i}\right\}$ be a collection of at least $2^{n}$ linear maps of $V$ to itself with $\operatorname{rk}\left(M_{i}\right)=\operatorname{ark}\left(M_{i}\right)=1$ for each $i$. Then there exist $i$ and $j$ with $i \neq j$ such that $M_{i}$ and $M_{j}$ are compatible.

Proof. We proceed by induction on the dimension $n$. When $n=1$, each $M_{i}$ is an isomorphism; so any pair is compatible. Thus, $\mathcal{M}$ need have only two elements for the conclusion of the lemma to hold. Now assume that the lemma is true for $n-1$. Assume that $M_{0} \in \mathcal{M}$ is not compatible with any other $M_{i}$ (otherwise, we are done). Since each element of $\mathcal{M}$ has rank 1, this means that for each $i \neq 0$, either $M_{i} M_{0}=0$ or $M_{0} M_{i}=0$. Define the sets $\mathcal{A}:=\left\{M_{i} \in \mathcal{M} \mid M_{0} M_{i}=0\right\}$ and $\mathcal{B}:=\left\{M_{i} \in \mathcal{M} \mid M_{i} M_{0}=0\right\}$. (Note that $\mathcal{A}$ and $\mathcal{B}$ are not necessarily disjoint.)

Consider the set $\mathcal{A}$. For each $M_{i} \in \mathcal{A}, \operatorname{im}\left(M_{i}\right) \subset \operatorname{ker}\left(M_{0}\right)$. Define $\widetilde{M}_{i}$ : $\operatorname{ker}\left(M_{0}\right) \rightarrow \operatorname{ker}\left(M_{0}\right)$ to be the restriction of $M_{i}$ to $\operatorname{ker}\left(M_{0}\right)$, and let $\widetilde{\mathcal{A}}=\left\{\widetilde{M}_{i} \mid M_{i} \in\right.$ $\mathcal{A}\}$ (note that $\left.\operatorname{dim}\left(\operatorname{ker}\left(M_{0}\right)\right)=n-1\right)$.

We claim that $\operatorname{rk}\left(\widetilde{M}_{i}\right)=\operatorname{ark}\left(\widetilde{M}_{i}\right)=1$ for each $\widetilde{M}_{i} \in \widetilde{\mathcal{A}}$. Since $\operatorname{rk}\left(\widetilde{M}_{i}\right) \leq \operatorname{rk}\left(M_{i}\right)=$ 1 , to prove the claim it suffices to show that $\widetilde{M}_{i}$ is nonnilpotent. If $\widetilde{M}_{i}=0$, then $\operatorname{ker}\left(M_{0}\right) \subset \operatorname{ker}\left(M_{i}\right)$, and, in fact, $\operatorname{ker}\left(M_{0}\right)=\operatorname{ker}\left(M_{i}\right)$ since both subspaces are of dimension $n-1$. But then $\operatorname{im}\left(M_{i}\right) \subset \operatorname{ker}\left(M_{0}\right)=\operatorname{ker}\left(M_{i}\right)$, so that $M_{i}^{2}=0$, contrary to hypothesis. Thus, $\widetilde{M}_{i} \neq 0$; so $M_{i}\left(\operatorname{ker}\left(M_{0}\right)\right)=\operatorname{im}\left(M_{i}\right) \subset \operatorname{ker}\left(M_{0}\right)$. Therefore, since $M_{i}$ is nonnilpotent, so is $\widetilde{M}_{i}$.

Now consider the set $\mathcal{B}$. For each $M_{i} \in \mathcal{B}, \operatorname{im}\left(M_{0}\right) \subset \operatorname{ker}\left(M_{i}\right)$. Thus if we let $\bar{V}=V / \operatorname{im}\left(M_{0}\right)$, the quotient map $\bar{M}_{i}: \bar{V} \rightarrow \bar{V}$ is well defined for each $M_{i} \in \mathcal{B}$. Let $\overline{\mathcal{B}}=\left\{\bar{M}_{i} \mid M_{i} \in \mathcal{B}\right\}$ (note that $\operatorname{dim}(\bar{V})=n-1$ ).

We claim that $\operatorname{rk}\left(\bar{M}_{i}\right)=\operatorname{ark}\left(\bar{M}_{i}\right)=1$ for each $\bar{M}_{i} \in \overline{\mathcal{B}}$. As was the case for $\widetilde{M}_{i}$, it suffices to show that $\bar{M}_{i}$ is nonnilpotent. If $\bar{M}_{i}=0$, then $\operatorname{im}\left(M_{i}\right)=$ $\operatorname{im}\left(M_{0}\right)$. But then $\operatorname{im}\left(M_{i}\right) \subset \operatorname{ker}\left(M_{i}\right)$; so $M_{i}^{2}=0$, contrary to hypothesis. Thus, $\operatorname{im}\left(M_{i}\right) \cap \operatorname{im}\left(M_{0}\right)=\{0\}$, and the fact that $M_{i}$ is nonnilpotent implies that $\bar{M}_{i}$ is as well.

Clearly, if $\widetilde{M}_{i}$ and $\widetilde{M}_{j}$ (or $\bar{M}_{i}$ and $\bar{M}_{j}$ ) are compatible, then so are $M_{i}$ and $M_{j}$ (since every matrix involved has rank 1). Thus we wish to apply the induction hypothesis to either $\widetilde{\mathcal{A}}$ or $\overline{\mathcal{B}}$. Since $\#(\widetilde{\mathcal{A}})=\#(\mathcal{A}), \#(\overline{\mathcal{B}})=\#(\mathcal{B}), \#(\mathcal{M}) \geq 2^{n}$, and $A \cup B=\mathcal{M} \backslash\left\{M_{0}\right\}$, we have that

$$
\#(\widetilde{\mathcal{A}})+\#(\overline{\mathcal{B}}) \geq \#(\mathcal{M})-1 \geq 2^{n}-1
$$

Thus either $\widetilde{\mathcal{A}}$ or $\overline{\mathcal{B}}$ has at least $2^{n-1}$ elements; so we can apply the induction hypothesis, completing the induction. 
Of course, if there are no nonnilpotent words, there can be no compatible pairs. Thus the simpler question of finding nonnilpotent words is of interest. Given two matrices $M_{1}$ and $M_{2}$, is there an upper bound on the shortest word $\omega$ such that $\left(M_{i}\right)_{\omega}$ is nonnilpotent? The $2 \times 2$ case has an easy answer.

Theorem 5.13. Let $M_{1}$ and $M_{2}$ be $2 \times 2$ matrices. If $\left(M_{i}\right)_{\omega}$ is nilpotent for every word $\omega$ of length less than or equal to two, then $\left(M_{i}\right)_{\alpha}$ is nilpotent for every finite word $\alpha$.

Proof. Note that a $2 \times 2$ matrix is nilpotent if and only if its square is zero. By assumption, the words $(1),(2),(1,2)$, and $(2,1)$ produce nilpotent matrices; so $(1,1),(2,2),(1,2,1,2)$, and $(2,1,2,1)$ produce the zero matrix. It is easy to see that if $\alpha$ is any finite word, then $2 \cdot \alpha$ must contain one of the latter four words as a subword. Thus $0=\left(M_{i}\right)_{(2 \cdot \alpha)}=\left(M_{i}\right)_{\alpha}^{2}$.

The problem seems to be harder in dimensions higher than two. We do have some partial answers, however. Recall that if $M$ is a nilpotent matrix, then $\operatorname{ind}(M)$, the index of $M$, is the least integer $k$ such that $M^{k}=0$ (see [8, $\left.\S 57\right]$ ).

Theorem 5.14 ([24]). Suppose that $M_{1}$ and $M_{2}$ are $n \times n$ matrices, $\operatorname{rk}\left(M_{1}\right)=$ $\operatorname{ind}\left(M_{1}\right)-1$, and $M_{1}^{s} M_{2}^{t}$ is nilpotent for all nonnegative integers $s$ and $t$ such that $0 \leq s+t \leq n$. Then $\left(M_{i}\right)_{\alpha}$ is nilpotent for any finite word $\alpha$.

Before we continue, we need to generalize our notation slightly. If $\left\{M_{i}\right\}_{i=1}^{n}$ is a collection of $k \times k$ matrices and $\omega=\left(\omega_{0}, \ldots, \omega_{l}\right) \in\{1,2, \ldots, n\}^{l+1}$, where $l$ is some positive integer (we call $\omega$ an $n$-word), then define the matrix $\left(M_{i}\right)_{\omega}$ to be

$$
\left(M_{i}\right)_{\omega}=M_{\omega_{l}} \circ M_{\omega_{l-1}} \circ \cdots \circ M_{\omega_{1}} \circ M_{\omega_{0}} .
$$

Also, if $\left\{\omega^{i}\right\}_{i=1}^{m}$ is a collection of $n$-words and $\beta=\left(\beta_{0}, \ldots, \beta_{p}\right) \in\{1, \ldots, m\}^{p+1}$, define the word $\beta\left(\omega^{1}, \ldots, \omega^{m}\right)$ to be $\left(\omega^{\beta_{0}}, \omega^{\beta_{1}}, \ldots, \omega^{\beta_{p}}\right)$.

Lemma 5.15 ([2, Cor. 2]). Let $\left\{M_{i}\right\}_{i=1}^{n}$ be a collection of $k \times k$ matrices. If $\left(M_{i}\right)_{\omega}$ is nilpotent for every $n$-word $\omega$ of length less than or equal to $k$, then any linear combination $\sum t_{i} M_{i}$ is also nilpotent.

Corollary 5.16. If $A$ (the $d \times d$ matrix representing the map $\left(f_{P}\right)_{q}: H_{q}\left(K_{L},[L]_{K_{L}}\right)$ $\left.\rightarrow H_{q}\left(K_{L},[L]_{K_{L}}\right)\right)$ is nonnilpotent, then there exists a word $\omega$ of length less than or equal to $d$ such that $\left(A_{*}\right)_{\omega}$ is nonnilpotent.

Proof. This follows immediately from the previous lemma and the fact that $A=$ $A_{1}+A_{2}$.

Remark 5.17. The word $\omega$ could of course be (1) or (2). However, if both $A_{1}$ and $A_{2}$ are nilpotent and $A$ is not, then the above theorem tells us that there is a nonnilpotent $\omega$ with $l(\omega) \leq d$ and $\omega \neq n \cdot(1)$ or $n \cdot(2)$ for any $n$. Thus there is a point in $S$ whose forward orbit intersects both $N_{1}$ and $N_{2}$ infinitely many times.

Corollary 5.18. Let $\Omega=\left\{\omega^{1}, \ldots, \omega^{k}\right\}$ be a set of distinct elements of $\{1,2\}^{l}$. Let $n=2^{l}-k$, and let $\mathcal{A}=\left\{\alpha^{1}, \ldots, \alpha^{n}\right\}=\{1,2\}^{l} \backslash \Omega$ (i.e., $\mathcal{A}$ consists of all the remaining words of length $l$ ). If the matrix $M:=A^{l}-\sum_{j=1}^{k}\left(A_{*}\right)_{\omega^{j}}$ is nonnilpotent, then there exist an integer $m, 0<m \leq d$, and $a \beta \in\{1, \ldots, n\}^{m}$ such that $\left(A_{*}\right)_{\beta\left(\alpha^{1}, \ldots, \alpha^{n}\right)}$ is nonnilpotent.

Proof. Since $A=A_{1}+A_{2}, A^{l}=\sum_{\gamma \in\{1,2\}^{l}}\left(A_{*}\right)_{\gamma}$. Thus, $M=\sum_{j=1}^{n}\left(A_{*}\right)_{\alpha^{j}}$, and we may apply Lemma 5.15 . 
For example, if $A^{l}-A_{1}^{l}-A_{2}^{l}$ is nonnilpotent, then there is a word $\omega$ of length less than or equal to $d l$ and containing both 1 's and 2 's such that $\left(A_{*}\right)_{\omega}$ is nonnilpotent.

Given a square matrix $A$, let $C(A)$ denote its characteristic polynomial.

Theorem 5.19. Let $\widetilde{A}=\left(\begin{array}{c|c}A_{11} & 0 \\ \hline 0 & A_{22}\end{array}\right)$. If $C(\widetilde{A}) \neq C(A)$, then there exist an integer $n, 2 \leq n \leq d$, and a word $\omega \in \Omega:=\{1,2\}^{n} \backslash\{n \cdot(1), n \cdot(2)\}$ such that $\left(A_{*}\right)_{\omega}$ is nonnilpotent.

Proof. Let $M$ be a $d \times d$ matrix, and $\operatorname{tr}(M)$ its trace. Recall that by Newton's formula, if we know $\operatorname{tr}\left(M^{j}\right)$ for $1 \leq j \leq d$, then we know $C(M)$, and vice versa $(6$. $\S 92])$. In particular, if $M$ is nilpotent, then $\operatorname{tr}\left(M^{m}\right)=0$ for all $m$.

Define the matrices $\widetilde{A}_{1}=\left(\begin{array}{c|c}A_{11} & 0 \\ \hline 0 & 0\end{array}\right)$ and $\widetilde{A}_{2}=\left(\begin{array}{l|c}0 & 0 \\ \hline 0 & A_{22}\end{array}\right)$, and observe that $\operatorname{tr}\left(\widetilde{A}_{i}^{m}\right)=\operatorname{tr}\left(A_{i}^{m}\right)$ for all $m$. Now, since $\operatorname{tr}(A)=\operatorname{tr}(\widetilde{A})$ and $A$ and $\widetilde{A}$ do not have the same characteristic polynomial, Newton's formula tells us that $\operatorname{tr}\left(A^{n}\right) \neq \operatorname{tr}\left(\widetilde{A}^{n}\right)$ for some $n, 2 \leq n \leq d$. It is clear that $\widetilde{A}^{n}=\widetilde{A}_{1}^{n}+\widetilde{A}_{2}^{n}$, and since $A=A_{1}+A_{2}$, we have that

$$
A^{n}=\sum_{\omega \in\{1,2\}^{n}}\left(A_{*}\right)_{\omega}=A_{1}^{n}+A_{2}^{n}+\sum_{\omega \in \Omega}\left(A_{*}\right)_{\omega} .
$$

Therefore, since trace is a linear function, we have

$$
\operatorname{tr}\left(A_{1}^{n}\right)+\operatorname{tr}\left(A_{2}^{n}\right)+\sum_{\omega \in \Omega} \operatorname{tr}\left(\left(A_{*}\right)_{\omega}\right) \neq \operatorname{tr}\left(\widetilde{A}_{1}^{n}\right)+\operatorname{tr}\left(\widetilde{A}_{2}^{n}\right) ;
$$

so $\sum_{\omega \in \Omega} \operatorname{tr}\left(\left(A_{*}\right)_{\omega}\right) \neq 0$. Therefore, $\operatorname{tr}\left(\left(A_{*}\right)_{\omega}\right) \neq 0$ for some $\omega \in \Omega$; so $\left(A_{*}\right)_{\omega}$ is nonnilpotent.

Lemma 5.20. If $\operatorname{rk}\left(A_{i}\right)=\operatorname{rk}\left(A_{i}^{2}\right)=1(i=1,2)$ and $\operatorname{tr}\left((\widetilde{A})^{2}\right) \neq \operatorname{tr}\left(A^{2}\right)$, then any finite product of $A_{1}$ and $A_{2}$ is nonnilpotent.

Proof. We have that $0 \neq \operatorname{tr}\left(A^{2}\right)-\operatorname{tr}\left((\widetilde{A})^{2}\right)=\operatorname{tr}\left(A_{1} A_{2}\right)+\operatorname{tr}\left(A_{2} A_{1}\right)$. Thus one of the matrix products on the right must have nonzero trace, and is therefore nonnilpotent. This means that both are nonnilpotent; so they must have rank one. Therefore, $A_{1}$ and $A_{2}$ are compatible, and the result follows from Lemma 5.2 .

Corollary 5.21. If, for some $q>0, \operatorname{rk}\left(A_{i}\right)=\operatorname{rk}\left(A_{i}^{2}\right)=1(i=1,2)$ and $\operatorname{tr}\left(A_{1}^{2}\right)+$ $\operatorname{tr}\left(A_{2}^{2}\right) \neq \operatorname{tr}\left(A^{2}\right)$ (where, as always, $A$ and $A_{i}$ are the Conley index homology maps from Definition 4.3), then $f: S \rightarrow S$ factors via $\rho$ onto the full two-shift.

Remark 5.22. We can easily generalize the results in this section in two directions. First, we can detect renewal systems generated by three or more words, not just two. Also, we can decompose the isolating neighborhood into three or more pieces. The only change that we need to make to most of the results to apply them to these cases is to generalize the definitions.

\section{Comparison to previous Results}

Other papers that use the Conley index to detect chaos in one form or another include [3], [4], [12, 13], 14], 15], 21], 23]. We conclude by comparing our method to several of these other approaches.

Our method is most similar to that developed by Szymczak in [21]. One difference is that his paper offers no way to detect whether two matrices will have all of their products nonnilpotent. This makes little difference in low dimensions, but it can 
be problematic when the matrices become large. Also, it considers only products of the $\left(A_{*}\right)_{\omega}$ 's (to use our notation). This, as noted in Theorem 5.8, can lead to computational inefficiencies and lost information.

Carbinatto, Kwapisz, and Mischaikow develop another approach to detecting chaos in [3]. Their result is essentially that if the characteristic polynomial of the matrix $A=\left(\begin{array}{l|l}A_{11} & A_{12} \\ \hline A_{21} & A_{22}\end{array}\right)$ differs in a certain way from that of the matrix $\widetilde{A}=\left(\begin{array}{c|c}A_{11} & 0 \\ \hline 0 & A_{22}\end{array}\right)$, then there exists a positive integer $n$ such that there is a semiconjugacy from $\left(S, f^{n}\right)$ onto $\left(\Sigma_{2}^{+}, \sigma\right)$. Similar results have been used to demonstrate the existence of chaos for the Henon map ([13]), the Lorenz attractor ([12, 14, 15]), and the Rössler equations ([28]).

A disadvantage of this approach is that it does not specify the integer $n$. This means, in particular, that it cannot be used to provide an entropy estimate (we know that $h_{t o p}(f) \geq \frac{\log 2}{n}$, but since $n$ is unknown, all we can conclude is that $f$ has positive entropy). (Our method can be used to show that $n=2$ for the Lorenz attractor, for example, by applying it to the matrices found in [12].)

There are also cases where this approach does not apply, but our method gives positive results. One can check that for

$$
A=\left(\begin{array}{cc|cc}
1 & 1 & 0 & 1 \\
1 & 1 & 0 & 2 \\
\hline-2 & 4 & 1 & 1 \\
-4 & 2 & 1 & 1
\end{array}\right),
$$

$A$ and $\widetilde{A}$ have the same characteristic polynomial. However, we can use Theorem 5.6 to show that in this case, $\rho(S)$ contains the renewal system generated by the words $(1)$ and $(1,2,1)$. Furthermore, an analysis of the proof of Theorem 1.1 of [3] shows that if its hypotheses are satisfied, our method will eventually detect a renewal system with positive entropy. The disadvantage is that we might have to spend a long time looking for it, while their theorem guarantees its existence immediately. (In [27] we present a version of the result from [3] which, under additional hypotheses, gives an upper bound for the integer $n$.)

It is worth noting again that Kwapisz in [9] studies the question of which products of a set of matrices are nonnilpotent in much greater depth and generality than we attempt here. He presents an algorithm for determining whether some power $f^{n}$ of the map factors onto the full 2-shift, but, as in [3], does not specify $n$.

A final strength of the method presented in this paper is the ease with which it can be generalized (see Remark 5.22 ).

\section{REFERENCES}

[1] Lluís Alsedà, Jaume Llibre, and Michał Misiurewicz, Combinatorial dynamics and entropy in dimension one, second ed., World Scientific Publishing Co. Inc., River Edge, NJ, 2000. MR 2001j:37073

[2] S. A. Amitsur, On the characteristic polynomial of a sum of matrices, Linear and Multilinear Algebra 8 (1979/80), no. 3, 177-182. MR 82a:15014

[3] Maria C. Carbinatto, Jaroslaw Kwapisz, and Konstantin Mischaikow, Horseshoes and the Conley index spectrum, Ergodic Theory Dynam. Systems 20 (2000), no. 2, 365-377. MR 2001e:37020

[4] Maria C. Carbinatto and Konstantin Mischaikow, Horseshoes and the Conley index spectrum. II. The theorem is sharp, Discrete Contin. Dynam. Systems 5 (1999), no. 3, 599-616. MR 2000i:37009 
[5] Charles Conley, Isolated invariant sets and the Morse index, CBMS Regional Conference Series in Mathematics, vol. 38, American Mathematical Society, Providence, RI, 1978. MR 80c:58009

[6] Leonard Eugene Dickson, New First Course in the Theory of Equations, John Wiley and Sons, Inc., New York, 1939. MR 1:1b

[7] John Franks and David Richeson, Shift equivalence and the Conley index, Trans. Amer. Math. Soc. 352 (2000), no. 7, 3305-3322. MR 2000j:37013

[8] Paul R. Halmos, Finite-dimensional vector spaces, D. Van Nostrand Co., Inc., PrincetonToronto-New York-London, 1958, 2nd ed., The University Series in Undergraduate Mathematics. MR 19:725b

[9] Jaroslaw Kwapisz, Cocyclic subshifts, Math. Z. 234 (2000), no. 2, 255-290. MR 2001j:37025

[10] Douglas Lind and Brian Marcus, An introduction to symbolic dynamics and coding, Cambridge University Press, Cambridge, 1995. MR 97a:58050

[11] Konstantin Mischaikow, The Conley index theory: a brief introduction, Conley index theory (Warsaw, 1997), Polish Acad. Sci., Warsaw, 1999, pp. 9-19. MR 2000c:37018

[12] Konstantin Mischaikow and Marian Mrozek, Chaos in the Lorenz equations: a computerassisted proof, Bull. Amer. Math. Soc. (N.S.) 32 (1995), no. 1, 66-72. MR 95e:58121

[13] _ Isolating neighborhoods and chaos, Japan J. Indust. Appl. Math. 12 (1995), no. 2, 205-236. MR 96e:58104

[14] _ Chaos in the Lorenz equations: a computer assisted proof. II. Details, Math. Comp. 67 (1998), no. 223, 1023-1046. MR 98m:58095

[15] Konstantin Mischaikow, Marian Mrozek, and Andrzej Szymczak, Chaos in the Lorenz equations: a computer assisted proof. III. Classical parameter values, J. Differential Equations 169 (2001), no. 1, 17-56, Special issue in celebration of Jack K. Hale's 70th birthday, Part 3 (Atlanta, GA/Lisbon, 1998).

[16] Marian Mrozek, Leray functor and cohomological Conley index for discrete dynamical systems, Trans. Amer. Math. Soc. 318 (1990), no. 1, 149-178. MR 90f:34076

[17] - The Conley index and rigorous numerics, Non-linear analysis and boundary value problems for ordinary differential equations (Udine), Springer, Vienna, 1996, pp. 175-195.

[18] David Richeson, private communication, 1998.

[19] Joel W. Robbin and Dietmar Salamon, Dynamical systems, shape theory and the Conley index, Ergodic Theory Dynam. Systems 8 (1988), Charles Conley Memorial Issue, 375-393. MR 89h:58094

[20] Dietmar Salamon, Connected simple systems and the Conley index of isolated invariant sets, Trans. Amer. Math. Soc. 291 (1985), no. 1, 1-41. MR 87e:58182

[21] Andrzej Szymczak, The Conley index for decompositions of isolated invariant sets, Fund. Math. 148 (1995), no. 1, 71-90. MR 96m:58154

[22] — The Conley index for discrete semidynamical systems, Topology Appl. 66 (1995), no. 3, 215-240. MR 97f:58113

[23] _ The Conley index and symbolic dynamics, Topology 35 (1996), no. 2, 287-299. MR 97b:58054

[24] V. A. Ufnarovskiı̌ and G. P. Chekanu, Nilpotent matrices, Mat. Issled. (1985), no. 85 Algebry, Koltsa i Topologii, 130-141, 155. MR 87e:15033

[25] Frank W. Warner, Foundations of differentiable manifolds and Lie groups, Springer-Verlag, New York, 1983. MR 84k:58001

[26] R. F. Williams, Classification of one dimensional attractors, Global Analysis (Proc. Sympos. Pure Math., Vol. XIV, Berkeley, Calif., 1968), Amer. Math. Soc., Providence, R.I., 1970, pp. 341-361. MR 42:1134

[27] Jim Wiseman, Symbolic dynamics from signed matrices, preprint, 2001.

[28] Piotr Zgliczyński, Computer assisted proof of chaos in the Rössler equations and in the Hénon map, Nonlinearity 10 (1997), no. 1, 243-252. MR 98g:58120

Northwestern University, Evanston, ILlinois 60208

Current address: Department of Mathematics and Statistics, Swarthmore College, 500 College Ave., Swarthmore, Pennsylvania 19081

E-mail address: jwisema1@swarthmore.edu 